\title{
N91-16932
}

\section{PROBING THE TIDES IN INTERACTING GALAXY PAIRS}

\author{
Kirk D, Borne \\ Space Telescope Science Institute \\ Baltimore, Maryland
}

\begin{abstract}
Detailed spectroscopic and imaging observations of colliding elliptical galaxies have revealed unmistakable diagnostic signatures of the tidal interactions. It is possible to compare both the distorted luminosity distributions and the disturbed internal rotation profiles with numerical simulations in order to model the strength of the tidal gravitational field acting within a given pair of galaxies. Using the best-fit numerical model, one can then measure directly the mass of a specific interacting binary system. This technique applies to individual pairs and therefore complements the classical methods of measuring the masses of galaxy pairs in well-defined statistical samples. The "personalized" modeling of galaxy pairs also permits the derivation of each binary's orbit, spatial orientation, and interaction timescale. Similarly, one can probe, the tides in less-detailed observations of disturbed galaxies in order to estimate some of the physical parameters for larger samples of interacting galaxy pairs. These parameters are useful inputs to the more universal problems of (1) the galaxy merger rate, (2) the strength and duration of the driving forces behind tidally-stimulated phenomena (e.g., starbursts and maybe QSOs), and (3) the identification of long-lived signatures of interaction/merger events.
\end{abstract}

\section{INTRODUCTION}

We have used the observed tidal disturbances in interacting galaxies to deduce various properties of the galaxies and of their relative orbits. The probes that we use to study these properties are the morphological and kinematic disturbances seen in the colliding galaxies. From these analyses, which employ a simple numerical simulation algorithm (Borne 1988a), we have been able to derive, for several interacting pairs, the galaxy masses, the internal properties of the galaxies (e.g., structure and rotation), the relative collision trajectory, the strength of the tidal encounter, and an age (or timescale) for the interaction (see Borne and Hoessel 1988; Borne 1988b; Borne, Balcells, and Hoessel 1988; and Balcells, Borne, and Hoessel 1989a,b). The most detailed analyses have been carried out for several pairs of interacting ellipticals identified in the Karachentsev (1972) catalog of isolated pairs; some of the results derived from "probing the tides" in these systems are described below (\$4). In addition, we describe the results of some simple studies of other interacting galaxies (\$§2-3), as well some preliminary results from a detailed study of the putative merger remnant NGC 7252 ( $\$ 5$; Borne and Richstone 1990). In every case but one we assume that gravity alone produces the observed tidal phenomena; the exception is in our simulations of the 3 C 278 radio jets seen in NGC $4782(\S 4[c]$ ). 


\section{THE DOUBLE-NUCLEUS CD GALAXY IN ABELL 2052}

The brightest galaxy in the rich cluster Abell 2052 is a double-nucleus $\mathrm{cD}$. The nuclei are separated by $7^{\prime \prime}$, having a relative velocity of $50 \mathrm{~km} \mathrm{~s}^{-1}$ (see Figs. 4 and 8 in Hoessel, Borne, and Schneider 1985). Kriss, Malumuth, and Borne (1990) have measured the radial variations of mean stellar velocity and velocity dispersion along the major axis of the primary galaxy, specifically excluding the small secondary nucleus. Their velocity profiles show that the velocity dispersion in the $\mathrm{cD}$ initially increases with radius in both directions, and then falls: ranging from $230 \mathrm{~km} \mathrm{~s}^{-1}$ at $R=0^{\prime \prime}$, up to $380 \mathrm{~km} \mathrm{~s}^{-1}$ at $|R| \approx 6^{\prime \prime}$, and down to $<200 \mathrm{~km} \mathrm{~s}^{-1}$ at $|R| \geq 12^{\prime \prime}$. The rotation profile also shows non-systematic variations at $|R| \approx 4-10^{\prime \prime}$.

We have run numerical simulations of this apparent cannibalism event, wherein a large galaxy accretes a small companion. We expected the size and shape of the velocity dispersion peaks at $|R| \approx 6^{\prime \prime}$ to vary with the assumed mass of the companion, but the actual results were surprising. We found that, as a result of the expected dynamic heating effect (i.e., the stirring of the $\mathrm{cD}$ by the companion), a large-mass companion produces a nearly flat projected velocity dispersion profile in the $\mathrm{cD}$. On the other hand, a low-mass companion does not have a significant dynamic effect on the dispersion profile, but it does have a pronounced kinematic effect in that the lower mass companion suffers more extensive stripping around its orbit, leading to significant superposition of its stripped starlight onto the starlight of the $\mathrm{cD}$. This produces the desired stellar velocity dispersion peaks at the location of the stripped stars (roughly at the orbital radius of the companion). Obviously, the mass of the companion cannot be so low as to produce statistically insignificant superposition. Hence, we were able to put rough constraints on the mass and orbit of the companion. These results are supported by the detection of increased surface brightness on one side of the $\mathrm{cD}$ ( $\mathrm{T}$. Lauer, private communication) and by a stream of gas, detected in $\mathrm{H} \alpha$, which wraps around the $\mathrm{cD}$ in such a way that one end of the stream coincides with the secondary nucleus (S. Baum, private communication).

Therefore, from the tidal effects seen in this $\mathrm{cD}$ galaxy, we conclude that the small secondary galaxy probably had intermediate to low mass (mass ratio $\approx 0.1$ ), and that stripping has already removed much of its mass (as evidenced by the $\mathrm{H} \alpha$ stream, the rotation anomalies and velocity dispersion peaks in the $\mathrm{cD}$, and the asymmetries in the $\mathrm{cD}$ surface brightness distribution).

\section{A MULTIPLE-RING GALAXY IN ABELL 2198}

Pence and Oegerle (1989) have discovered a peculiar double ring galaxy in the rich cluster Abell 2199. The galaxy shows two complete non-concentric rings, with radii of 4.5 and $7.8 \mathrm{kpc}$ $\left(H_{0}=100 \mathrm{~km} \mathrm{~s}^{-1} \mathrm{Mpc}^{-1}\right)$, and possibly a third incomplete ring (i.e., an arc) with a radius of $15 \mathrm{kpc}$. This galaxy is in a dense cluster environment, having several nearby companions (in projection). Pence, Oegerle, and Borne (1990) describe models for the formation of these multiple rings through deeply-penetrating collisions of massive companions into target disk galaxies. We found that the number of rings and the relative ring diameters vary with the mass of the impinging satellite: a high-mass satellite (mass ratio $\approx 0.4$ ) produces many complete 
rings, with a large spacing between the two outermost rings, while a lower mass satellite (mass ratio $\approx 0.1$ ) produces fewer rings, most of which are incomplete (i.e., arcs), with a smaller relative spacing between the two outermost rings. Given the near completeness of the rings observed in the Abell 2199 multiple-ring galaxy and given their relatively close spacing, we conclude that if the rings are in fact collisionally-induced, then the mass of the ring-producing companion is somewhere between the two extremes mentioned (i.e., mass ratio $\approx 0.2-0.3$ ).

\section{DISTORTED E-E PAIRS}

We have been involved for several years in analyzing the physical properties of strongly interacting pairs of elliptical galaxies (Borne 1988a,b; Borne and Hoessel-1988; Borne, Balcells, and Hoessel 1988; and Balcells, Borne, and Hoessel 1989a,b). We have been able to use the tidal disturbances observed both in morphological data and in positional velocity data to constrain collision models for the interacting pairs. From these models we have derived masses, orbits, spatial orientations, and interaction timescales. Among the tidal features that we have matched with our simulations are: the asymmetric light distributions, the U-shaped rotation profiles (Borne and Hoessel 1988; Borne 1990), and the heated velocity dispersion profiles. Though it is not possible to completely disentangle the two effects, it is found that the morphological distortions help to constrain the relative orbit of the galaxies, while the kinematic disturbances constrain the age and strength of the collision. Taken together, all of the observed distortions correlate with the strength and the active lifetime of the tidal potential field.

It should be possible to apply the results of our simulations of specific pairs to determinations of the age and duration of interactions in a much larger sample of tidally-disturbed galaxies. For example, the smallest radius at which the symmetry of the galaxy is broken due to tides corresponds roughly to the radius at which the time for orbital mixing (i.e., a few orbital periods) equals the time since maximum tidal impulse. Such age-dating can be calibrated using the several pairs for which we have already derived specific collision parameters.

We describe very briefly below a few of the physical results that were derived from our bestfit simulations of the tidal distortions seen in three particular pairs of interacting ellipticals.

\section{(a) NGC 1587/1588 = Karachentsev 90}

The tidal distortions observed in the surface brightness distribution of the primary galaxy constrain the mass of the smaller secondary galaxy: if $M / L$ were smaller for the secondary galaxy than for the primary galaxy (as suggested by the small velocity dispersion of the secondary; Borne and Hoessel 1988), then there would be almost no tidal distortion in the larger galaxy; if $M / L$ were larger for the secondary, then the predicted tidal distortions in the primary would be stronger than those actually observed; but if $M / L$ were the same for the two galaxies, then the simulated tidal distortions in the primary galaxy can be made to agree with those observed. As a result, we found that the mass ratio = luminosity ratio $=1 / 3$ (Borne $1988 b$ ):

In K99, as well as in general collisions between other hot stellar systems, the position angles of the tidally-compressed and tidally-distended isophotes correlate with the direction of the 
galaxies' motions: particles that are tidally stripped from hot stellar systems tend to trail the galaxy (Borne and Hoessel 1988; Borne 1990). In addition, we find that the narrowness of the tidal distention is directly related to the coldness of the internal stellar velocity distribution. We see this in the thin tidal tails emanating from strongly-rotating galaxies. In those cases, however, the direction of the tail does not correlate as well with the galaxy's orbital motion.

(b) NGC 2672/2673 = Karachentsev 175

We found that the tidal tails associated with the small galaxy were very difficult to reproduce (Balcells, Borne, and Hoessel 1989a,b), until we realized that the observed directions of the tails (extending to the north and to the east) required that the smaller galaxy be strongly rotating, even though we had no data either to support or to refute this claim. It was also found that the two orthogonal tails required the small galaxy to have passed partially through the core of the primary galaxy, in a reverse-tide regime, where $M(r)$ grows faster than $r^{2}$ (i.e., density falls more slowly than $1 / r$ ). Tidal distortions can therefore probe the internal kinematics and structure of colliding galaxies, leading to testable predictions about these physical parameters.

(c) NGC $4782 / 4783=3$ C 278

The large central stellar velocity dispersions observed in these galaxies (i.e., $\sigma_{4782} \approx 390$ $\mathrm{km} \mathrm{s}^{-1} ; \sigma_{4783} \approx 300 \mathrm{~km} \mathrm{~s}^{-1}$ ) appear to be evidence for strong nuclear tidal shocks that were induced by a deeply-penetrating collision between the two galaxies (Borne, Balcells, and Hoessel 1988; Balcells, Borne, and Hoessel 1989b). The strong nuclear tidal shock dumps a lot of matter into the cores of the galaxies, which could trigger some nuclear activity (i.e., "feed the monster"). This may be the cause of the radio jets (3C 278) seen emanating from NGC 4782, the galaxy with the largest velocity dispersion (i.e., the strongest tidal shock). Ballistic models of the radio jets (including both gravitational and ram pressure deflection; Borne and Colina 1990) indicate that the strong bends in the jets (particularly in the east jet) are not consistent with the galaxy motions required by our best-fit collision simulation (which was derived from the tidal distortions observed in optical light). In particular, it is not clear why the jet on the trailing (east) side of NGC 4782 should be bent so strongly and so much closer to the nucleus than the jet on the leading (west) edge, but it may relate to strong inhomogeneities in the spatial and velocity distributions of the ISM into which the jets are ejected. In this case, therefore, the observed tidal distortions have allowed us to test and make predictions about conditions related to the generation, propagation, and environments of extragalactic radio jets.

\section{THE MERGER REMNANT NGC 7252}

Borne and Richstone (1990) have examined the properties of the merger remnants from a large number of disk+disk collision simulations in an attempt to find a model for the claimed merger candidate NGC 7252 (Schweizer 1982). A set of orbit and disk galaxy parameters were found that indeed led to the desired final product (i.e., we were able to reproduce most of the peculiar morphological and kinematic features seen in this "train wreck"). Among the many useful results of this exercise (besides demonstrating that it is possible, in at least one instance, 
to make an elliptical-looking galaxy from the merger of two spirals) was the determination of a dynamical age for the system. In essence, the lengths and speeds of the tidal tails can be used to measure the time since the beginning of the interaction $\left(8-10 \times 10^{8}\right.$ years $)$. Our derived age agrees with that estimated by Schweizer (1982) both from the tail lengths $\left(9-10 \times 10^{8}\right.$ years) and from the blue colors and starburst spectrum $\left(4-15 \times 10^{8}\right.$ years $)$.

\section{SUMMARY}

We have presented results from several studies in which the tidal distortions observed in specific interacting and merging galaxies have been used to probe the dynamical properties of the galaxies (e.g., masses, rotation, relative orbits, interaction ages). This type of analysis can be extended to a much wider variety of colliding, interacting, merging, and merged galaxies, each with its own set of peculiar tidal features. The character of the observed tidal disturbances helps to identify real cases of interaction, as opposed to cases of simple projection. Examples of tidally-induced features include: (1) asymmetric morphology (e.g., loops, tails, bridges, rings), (2) peculiar velocity fields (e.g., enhanced velocity dispersions, U-shaped rotation in ellipticals, tidal shocks, one-sided $21 \mathrm{~cm}$ HI profiles in spirals; see Borne 1990), (3) unusual spectral features (e.g., signatures of a starburst stellar population, blue elliptical colors), and (4) bent radio jets.

While case studies of specific interacting pairs can be very rewarding and informative, analyses of large samples of such pairs are needed to answer the global questions about the frequency and significance of interactions and mergers among galaxies in the universe. These remain among the most fundamental unresolved questions related to paired and interacting galaxies.

\section{REFERENCES}

Balcells, M., Borne, K. D., and Hoessel, J. G. 1989a, Ap. J., 336, 655. . 1989b, Ap. Space Sci., 156, 215.

Borne, K. D. 1988a, Ap. J., 330, 38. . 1988b, Ap. J., 330, 61 . . 1990, in Dynamics and Interactions of Galaxies, ed. R. Wielen (Berlin: Springer), in press.

Borne, K. D., Balcells, M., and Hoessel, J. G. 1988, Ap. J., 333, 567.

Borne, K. D., and Colina, L. 1990, Ap. J., submitted.

Borne, K. D., and Hoessel, J. G. 1988, Ap. J., 330, 51.

Borne, K. D., and Richstone, D. O. 1990, Ap. J., submitted.

Hoessel, J. G., Borne, K. D., and Schneider, D. P. 1985, Ap. J., 293, 94.

Karachentsev, I. D. 1972, Comm. Spec. Astrophys. Obs. USSR, 7, 3.

Kriss, G. A., Malumuth, E. M., and Borne, K. D. 1990, in preparation.

Pence, W., and Oegerle, W. 1989, Bull. AAS, 21, 1170.

Pence, W., Oegerle, W., and Borne, K. D. 1990, in preparation.

Schweizer, F. 1982, Ap. J., 252, 455. 